\title{
Social distribution of internal exposure to environmental pollution in Flemish adolescents
}

Bert Morrens ${ }^{1 \S}$, Liesbeth Bruckers ${ }^{2}$, Elly Den Hond ${ }^{3}$, Vera Nelen ${ }^{4}$, Greet Schoeters ${ }^{3,7}$, Willy Baeyens ${ }^{5}$, Nicolas Van Larebeke ${ }^{6}$, Hans Keune ${ }^{1}$, Maaike Bilau ${ }^{8}$, Ilse Loots ${ }^{1}$

${ }^{1}$ Faculty of Political and Social Sciences, Department of Sociology, University of Antwerp, Belgium

${ }^{2}$ Interuniversity Institute for Biostatistics and statistical Bioinformatics, Hasselt University,

Diepenbeek-Belgium

${ }^{3}$ Department of Environmental Toxicology, Flemish Institute for Technological Research, Mol-

Belgium

${ }^{4}$ Provincial Institute of Hygiene, Antwerp-Belgium

${ }^{5}$ Department of Analytical and Environmental Chemistry, Brussels Free University, Belgium

${ }^{6}$ Department of Radiotherapy and Nuclear Medicine, University Ghent, Belgium

${ }^{7}$ Department of Biomedical Sciences, University of Antwerp, Belgium

${ }^{8}$ Department of Public Health, University Ghent, Belgium

${ }^{\S}$ Corresponding author

Bert Morrens

Sint Jacobstraat 2, B - 2000 Antwerpen

Tel: +32-3-265.55.40

E-mail addresses:

BM: bert.morrens@ua.ac.be

LB: liesbeth.bruckers@uhasselt.be

EDH: elly.denhond@vito.be

VN: vera.nelen@pih.provant.be

GS: greet.schoeters@vito.be

WB: wbaeyens@vub.ac.be

NVL: nicolas.vanlarebeke@ugent.be

HK : hans.keune@ua.ac.be

MB: M.Bilau@arcadisbelgium.be

IL: ilse.loots@ua.ac.be 


\section{Abstract}

Background: Environmental justice research suggests that inequalities in the distribution of environmental exposure to chemical pollution systematically disadvantage the lower social strata of society. The effects of these inequalities on the human exposure to pollution are however to a large extend unknown. The purpose of this study is to assess social gradients in human biomonitoring results of a representative sample of Flemish adolescents.

Methods: We investigate the associations between individual socioeconomic status (SES), measured by parental educational attainments, and internal body concentration of seven chemical compounds in biological samples of 1642 adolescents aged 14-15 in Flanders (Belgium): PCB's, HCB, DDE, lead, cadmium, benzene and PAHs. Social gradients in average and high exposure to these biomarkers were examined with geometric means and odds ratios (with 95\% confidence intervals), using multiple regression models, controlling for covariates and confounders.

Results: Depending on the (type of) pollutant, adolescents with a lower SES either have higher or lower internal concentrations. Chlorinated compounds (PCBs and pesticides HCB and DDE) are positively associated with SES (higher exposures for higher SES), while heavy metals (lead and cadmium) are negatively associated (higher exposures for lower SES). For metabolites of organic compounds (benzene and PAHs) we find no association with SES. Socially constructed factors, such as dietary and lifestyle habits, play an important role in these relations.

Conclusions: Our study suggests that the association between individual SES and the internal body concentration of exposure to environmental pollutants in Flemish adolescents is more complex than can be assumed on the basis of the environmental justice hypothesis.

\section{Keywords}

environmental justice; human biomonitoring; socioeconomic status; adolescents; internal environmental exposure 


\section{Background}

Since the last few decades, the field of public health has emphasized environmental and social factors as important, but largely separate, determinants of social health inequalities. Important because they both have proven to have damaging effects on the health status of people, separate because they are perceived as two different influences, needing different scientific and political expertise to monitor, evaluate and tackle them. Studies on the social determinants of health rarely include biological measurements of the chemical environment, and vice versa (Adler and Newman, 2002; O'Neill et al., 2003; Porta et al., 2008). Recently however, the interaction between environmental and social factors in producing health inequalities is becoming more and more visible as issues of poverty and health are increasingly entwined with environmental issues emerging out of technological development, global warming and persistent chemicals (O'Neill et al., 2007). Therefore, social and environmental epidemiologists underline the synergies between the biochemical environment and the social environment and stress the importance of exploring the role of environmental pollution as a contributing factor in health disparities (Evans and Kantrowitz, 2002; Krieger, 2001; O'Neill et al., 2007). This research is rooted in the environmental justice movement, which emerged more than two decades ago out of scientific and societal attention for poor AfricanAmerican communities in the US (Cole and Foster, 2001). The empirical grounding of this environmental justice movement shows that hazards in the physical and chemical environment disproportionately affect those individuals, households and neighbourhoods that also face hazards in their social environment (Brown, 1995).

Two potential disparities are distinguished, that can work independently or together. Minorities and socially disadvantaged populations can be more exposed to environmental pollution (proximity inequality) and can be more susceptible to its damaging health effects (impact inequality), while having a smaller contribution to environmental pressure. The socio-spatial distribution of environmental quality reveals that poor people and deprived communities may experience an accumulation of exposure to multiple, suboptimal environmental conditions (Evans and Kantrowitz, 2002) like proximity to hazardous waste facilities and busy roads, flood risk and air quality (Hipp and Lakon, 2010; Mitchell and Dorling, 2003; Walker and Burningham, 2011). Given the same exposure, disadvantaged groups may also be more vulnerable to adverse health effects than the general population. Due to several 'susceptibility factors' (Sexton, 1997) like predisposed diseases and psychological stress, the health impact of environmental pollution appeared to be greater in the lower social classes (Pope et al., 2002; Wheeler and Ben-Shlomo, 2005). 
Children and adolescents living in deprived households and neighbourhoods are identified as a particularly susceptible subgroup because their specific behavioural and dietary habits and their physical and mental development makes them even more vulnerable to adverse influences from the living environment (Bolte and Kohlhuber, 2005; Hornberg and Pauli, 2007).

These two types of inequality can independently affect a person's health status, yet those who are both more exposed and more susceptible will endure the most health effects. This combined inequality is sometimes referred to as the 'double jeopardy' of environmental health disparities (Morello-Frosch and Shenassa, 2006). Although it is clear that the combination of both types of inequality will be concentrated in the most deprived segment of a population, the relationship between SES and environmental health may be not confined to poor people alone. Health status follows a clear social gradient, meaning that adverse health outcomes get worse at every step down the social ladder (Wilkinson and Marmot, 2003).

Although the policy implications of the environmental justice discourse are far-reaching, the empirical grounding of this discourse has recently been criticised for using simplistic methodologies and aggregated data which give only a very partial view of the processes involved in environmental inequalities (Briggs et al., 2008; Walker, 2009). Research outside the US is also rather scarce, which raises questions about the validity of US-based results in other settings. Many environmental justice studies link the spatial distribution of pollution sources or emission concentrations to aggregated socio-demographic characteristics of neighbourhoods or city wards. However, the usefulness of this measurement seems to be rather limited, because analysed group-level associations between social determinants and (potential) external exposure to pollutants may not reflect the individual experience. Little research exists on the social distribution of internal pollutant exposure concentrations, measured by human biomonitoring, and available evidence shows inconsistent results which do not always support the environmental justice hypothesis (Borrell et al., 2004; Porta et al., 2008; Vrijheid et al., 2010).

In this paper we attempt to tackle this research gap by socio-stratifying the results of a human biomonitoring study in Flanders, the northern part of Belgium. Human biomonitoring is a technique for measuring the internal exposure and biological effects of chemical compounds in human tissue. Biomarkers of exposure indicate the amount of pollution actually absorbed and retained within the body. The majority of human biomonitoring studies, however, only use socioeconomic measures as 
confounding factors that 'control' for their potential 'disturbing' effects, and seldom analyse the specific relationship between socioeconomic indicators and biomarkers.

We investigate the associations between socioeconomic status (SES) and internal exposure to seven selected pollutants in Flemish adolescents aged 14-15. We explore whether specific social gradients emerge when assessing the internal concentration of pollutants in the human body, and whether these gradients can be explained by specific underlying factors related to both exposure and SES.

\section{Materials and methods}

The Flemish Centre for Environment and Health carried out the first Flemish Environment and health Survey (FLEHS I), a large-scale biomonitoring program on neonates, adolescents and older adults, between 2002 and 2006 in Flanders, the northern part of Belgium. The objectives were to measure and compare internal exposure to pollutants of people living in different geographical areas and to assess whether observed differences were associated with biological and health effects. Detailed results about the geographical distribution of the internal exposure to environmental pollution (and the related health effects) are described elsewhere (Croes et al., 2009; Den Hond et al., 2009; Schroijen et al., 2008). In this article we focus on the social distribution of the internal exposures among adolescents.

\section{Study area and subjects}

The human biomonitoring campaign of adolescents was carried out in eight areas of Flanders with a different pollution pressure: the Antwerp agglomeration, the Ghent agglomeration, the aggregated industrial harbour areas of Antwerp and Ghent (mainly petrochemical and metallurgic industries, respectively), the industrial zone around the Albert canal (chemical industry), the industrial zone of Olen (non-ferrous industry), the immediate surroundings of household waste incinerators, a rural area with intensive fruit cultivation, and a rural area (countryside). The total surface of the study site covers $22 \%$ of the total surface of Flanders and is inhabited by $20 \%$ of the Flemish populations.

The adolescents were enrolled via 50 schools located in the eight selected regions, and sampled between October 2003 and July 2004. Inclusion criteria were the following: being born in 1988 or 1989, studying in the third year of secondary education, living for at least five years in the same study area, and giving informed consent (both adolescent and parents). 4386 pupils were invited to participate in the human biomonitoring program by letter. 1670 (38.1\%) did not respond, because 
they did not fulfill the inclusion criteria or because they were not interested. Among the 2716 pupils who did respond, $646(23.8 \%)$ refused to participate and $138(5.0 \%)$ were excluded by the researchers because they did not reside in the area for 5 years or because of incomplete questionnaires or insufficient blood or urine sampled. The recruitment resulted in a total of 1679 adolescents, distributed equally over the eight study areas. All participants signed an informed consent form and had the right to withdraw from the study at any time. The study was approved by the Medical Ethics Committee of the University of Antwerp.

\section{Measures of internal exposure}

About $200 \mathrm{ml}$ of urine and $40 \mathrm{ml}$ of blood were sampled from each participant to carry out various chemical analyses and assess the internal concentration of 7 biomarkers of exposure, classified within three types of pollutants: chlorinated persistent compounds, heavy metals and volatile compounds. In the selection of these 7 biomarkers, priority was given to historical pollutants to which a general population may be chronically exposed through the environment or diet. These biomarkers are selected because they have well-known health effects, since levels in the general population are expected to be measurable and validated detection methods are available.

Three chlorinated persistent compounds were measured in serum: concentration of polychlorinated biphenyls (PCBs) (sum of marker PCB 138, 153 and 180) and concentration of the organochlorine pesticides hexachlorobenzene $(H C B)$ and $p, p^{\prime}$-dichlorodiphenyldichloroethylene ( $p, p^{\prime}-D D E, a$ metabolite of DDT). PCBs were industrially used since the 1950s as hydraulic or transformer fluids, as plasticisers in paint, and in carbonless copying paper. HCB and DDT were used to control insects and fungus. 2) Two heavy metals were measured in whole blood: lead and cadmium. In the past, they were mainly emitted by the non-ferro industry. Lead can also be found in leaded petrol and paint, cadmium is also present in tobacco smoke and can be emitted by waste incinerators. 3) Two metabolites of volatile compounds - PAHs (1-hydroxypyrene) and benzene ( $t, t^{\prime}$-muconic acid) were measured in urine. These compounds are associated with incomplete combustion or by traffic. The toxicological procedures of the blood and urine analyses are described in detail elsewhere (Schroijen et al., 2008).

\section{Measures of socioeconomic status}

Additionally to the collection of blood and urine, participants were asked to fill out questionnaires about personal, social, and lifestyle factors. Complementary, the parents of the adolescents completed a questionnaire comprising data on their education, housing, residence history, household income, and the health history of their participating child. Since our research subjects are 
14-15 year old adolescents, and thus not yet active on the labour market, their SES must be defined by parental or household indicators. We used parental educational attainment, defined as the highest educational level of the father and mother, to quantify the adolescent's SES. The questionnaire contains a standard scale with eight response categories representing the different stages of the Flemish education system. We used the highest educational level of one parent if the other parent's information was not listed. Adolescents living in co-parenting households had the opportunity to led parents fill out the education attainment for two separate households (household $A$ and $B$ ), and indicating the time the adolescent spend in each household. We classified parental educational attainment into three categories: primary, secondary and tertiary education, corresponding respectively to adolescents with a low, middle or high SES.

\section{Statistical methodology}

For each biomarker, two measures of pollutant concentration were examined in relation to SES: the average and high exposure concentration. Average exposure is quantified by the geometric mean (GM) for each category of SES. An adolescent is defined to have a high internal exposure if its individual exposure is higher than the $90^{\text {th }}$ percentile concentration (P90) of the entire group. Since $90 \%$ of all adolescents have a body concentration below this value, the P90 stipulates the peak value of the study sample for each biomarker. So, high exposure is defined as an internal exposure above the sample $90^{\text {th }}$ percentile. When associations or gradients between SES and biomarkers of exposure are 'positive', both variables move in tandem: an increase in SES correlates with an increase in exposure, and vice versa. When associations are 'negative', an increase in one variable goes together with a decrease in the second one.

Our analyses consist of (three) consecutive linear and logistic regression models. The first model investigates social gradients in the unadjusted geometric mean exposure ( $\mathrm{GM}$ and $95 \% \mathrm{Cl}$ ) and in the probability to have high exposure $(\mathrm{OR}$ and $95 \% \mathrm{Cl})$. Model 2 extends model 1 by including confounders to be known from literature to influence internal exposure: sex, age, smoking status and living region. For the chlorinated compounds also body mass index is included in this model. Correcting for these covariates may explain the initially found relations with SES. A third model additionally includes biomarker specific covariates. All analyses are done at a $5 \%$ level of significance with SAS version 9.1 and SPSS version 18.

\section{Results}


Using highest parental education as proxy for socioeconomic status (SES), we included 1642 adolescents in our study (2.2\% missing). 15,5\% of the adolescents have a low SES (meaning that both parents are primary educated), 34,9\% have a SES which we termed 'middle' (secondary education as highest attainment of parents), and $49,9 \%$ have a high SES (at least one parent with tertiary education).

Social distribution of indicators associated with exposure

Insert Table 1

Sex, age and BMI are significantly related to the SES of adolescents in our study sample. Age and BMI follow a negative social gradient (higher age and BMI for lower SES category). More boys are present in the lower SES categories (potentially indicating a selection bias). Lifestyle factors such as smoking, alcohol consumption and drug use also follow a significant negative social gradient. Daily tobacco smoking is reported three times more often by low compared to high SES adolescents. Our study sample however reveals no significant social gradients for the consumption of home grown vegetables or daily fish consumption. The daily consumption of meat however follows a significant negative gradient: lower SES consume more meat. Being breastfed as baby follows a positive social gradient: adolescents with high SES (tertiary educated parents) were significantly more breastfed than adolescents with lower SES. Adolescents with low SES reported higher exposure to environmental tobacco smoke (mean hours per day in locations where people smoke), but indicate less indoor use of pesticides. They also live more closely to busy traffic roads. In our study sample, adolescents with lower SES more frequently live in industrial areas, adolescents with high SES live more in rural areas. Low SES adolescents significantly report worse health status and more doctordiagnosed asthma.

\section{Insert Table 2}

\section{Social distribution of biomarkers of internal exposure}

In table 3 model 1 shows the unadjusted geometric mean and the probability of having a high concentration to chlorinated compounds. For PCBs the mean exposure significantly increases with increasing SES. Also the odds for high exposure (>P90) follows a significant and positive gradient. Sex, age, smoking status and BMI are important determining factors of the internal concentration of PCBs and these factors are significantly associated with SES. However model 2 shows that controlling for 
these factors does not diminish the effect of SES. Further correction for breastfeeding in model 3 results in a non significant social gradient for high exposure. The OR for the low and middle SES category approach 1 (indicating no difference with the highest category) and are no longer statistically significant. The social gradient for the risk of having high PCB exposure levels (>P90) in 14-15 year adolescents, can - at least partly - be explained by the social gradient in nursing with maternal milk when these adolescents were baby's. The positive social gradient in average exposure to PCBs however stays significant.

For $\mathrm{HCB}$ and $\mathrm{DDE}$, the unadjusted geometric mean concentration in model 1 shows a significant positive gradient with SES, but a non significant OR for high exposure risk. The positive gradient in geometric mean stays significant after correcting for sex, age, smoking status and BMI. Further correcting for breastfeeding explains the SES gradient in geometric mean for DDE but not for HCB.

Additionally adjusting all chlorinated compounds in model 3 for maternal age and parity (not in table) did not change our findings. No significant interactions with residential area or sex were found, meaning that social gradients in internal exposure to chlorinated compound did not differ between living areas or between sexes (results not shown).

\section{Insert Table 3}

For cadmium, we found a negative social gradient in the distribution of mean and high exposure. This is reflected in OR larger than 1 for the adolescents in the lower SES categories (model 1). The odds for high exposure of adolescents with low SES is more than double the odds for adolescents with high SES. The negative gradient in the mean and high exposure is still present after correction for sex, age, smoking status and study area in (model 2), and remains present after additionally correcting for the consumption of home grown vegetables (model 3). Note that SES is borderline not significant in model 2 for high exposure $(p=0.053)$. For lead we see a modest and non significant relation between SES and mean exposure, but a stronger and significant relation with high exposure. This effect disappears after correcting for sex, age, smoking status and living region.

Additionally testing our data for possible interaction of (1) SES and living area and (2) SES and gender resulted in a significant interaction effect between SES and living area for cadmium and between SES and sex for lead. The social dispersion in internal cadmium exposure significantly differs between living areas. In all, but one, areas adolescents with a low SES are more exposed than adolescents with a high SES. The difference in mean cadmium exposure, between low and high SES, is largest in 
the industrial region of Olen and smallest in the rural area. In the urban area of Ghent there appeared to be a converted social effect: the cadmium concentration of adolescents with a low SES is on average 0.53 times lower than the concentration of adolescents with a high SES.

The social distribution of lead exposure significantly interacts with gender. For boys, the average lead concentration in the lowest SES category equals 1.14 times the concentration in the highest SES, whereas for girls the average lead concentration in the lowest SES category equals 0.87 times the concentration in the highest category. This indicates that socioeconomic status has an opposite effect on lead concentration in boys and girls.

\section{Insert Table 4}

For the metabolites of organic compounds no social gradients were detected; not in terms of the geometric mean and not in high exposure (Table 4). No interactions with residential area or sex were found.

\section{Insert Table 5}

\section{Discussion}

In this study, we tested the general environmental justice hypothesis that people of lower socioeconomic strata are more exposed to environmental pollution than people of higher social strata. Our results show that the association between socioeconomic status (SES) and the internal body concentration of exposure to environmental pollutants in Flemish adolescents (aged 14-15) is more complex than can be assumed on the basis of the environmental justice hypothesis. Depending on the (type of) pollutant, adolescents with a lower (SES) have higher or lower internal concentrations. Chlorinated compounds (PCBs and pesticides HCB and DDE) are positively associated with SES, while heavy metals (lead and cadmium) are negatively associated. For metabolites of volatile compounds (benzene and PAHs) we find no association with SES. Further, our results show that socially constructed lifestyle factors and dietary habits play an important role in these relations. The positive relation between SES and PCBs (OR) or DDE (geometric mean) (higher SES have higher exposure) could not be explained by differences in sex, age, smoking, BMI or residential area, but disappeared after additional adjustment for breastfeeding. This indicates that the nursing habit of the mother is a principal factor in the observed social differences in the body concentration of PCBs and DDE among adolescents. The negative relation between SES and cadmium or lead (higher SES 
have lower exposure) could to a large extend by explained by age, sex, smoking behaviour and residential area of the adolescent.

Mean and high exposure to environmental pollutants were calculated after stratification of the participants by social class. From a toxicological point of view, analyzing high exposures is sometimes more important than modal or mean exposure, since most health risks are expected for persons and groups having the highest exposure. Similar analyses using $75^{\text {th }}$ or $95^{\text {th }}$ percentile concentrations to assess socioeconomic differences in internal exposure were conducted by other authors (Axelrad et al., 2009; Vrijheid et al., 2010). Our study suggests that when looking at the internal exposure of environmental chemicals, a complex and nuanced picture emerges in which social gradients differ in function of the chemicals under study. Our findings support only partly the environmental justice hypothesis, but the results are consistent with other available studies. Positive associations between indicators of SES and chlorinated compounds are also found elsewhere. In pregnant women, increasing income was associated with increasing concentrations of PCBs but not DDE in the US (Borrell et al., 2004), and with PCBs and HCB, but not DDE in Spain (Vrijheid et al., 2010). The PCB and DDE level of 4 year old children in the Michigan region (US) was positively associated with family SES (Jacobson et al., 1989). In Germany, PCBs, DDE and HCB concentrations were also higher in children aged 3 to 14 with a higher SES (Becker et al., 2008). However, not all studies are consistent with these findings. In a small study population of adults with pancreatic cancer in Spain, serum concentration of seven organochlorine compounds, including PCBs, $\mathrm{HCB}$ en DDE, were significantly higher for lower occupational social classes (Porta et al., 2008). In the Czech adult population, PCB concentrations were not significantly associated with individual educational attainment (Cerna et al., 2008). With respect to DDE, some American studies have suggested that there is a racial difference in DDE levels, with the highest levels being among African Americans (Davies et al., 1972). Social differences in the general or age specific populations are however mostly non significant, suggesting perhaps more cultural and ethnic than socioeconomic mechanisms behind unequal distributions of DDE concentrations.

PCBs, DDE and HCB have hormone-disrupting, immune-disrupting, neurotoxic and carcinogenic properties (Demers et al., 2002; Osius et al., 1999). Significant relations between these health effects and exposure concentrations of chlorinated compounds were found in a general population in Belgium (Den Hond et al., 2002; Staessen et al., 2001; Van den Heuvell et al., 2002; Vermeir et al., 2005). A higher fish and meat consumption and more nursing with maternal milk are identified as main determinants explaining the social differences in serum concentrations of chlorinated compounds. Similar to our findings, the initially found relation between education and PCBs in US 
children (4 years) disappeared after adjustment for breastfeeding (Jacobson et al., 1989). Pediatric studies in Europe, US, Canada and Australia have shown clear social class differences in the initiation and duration of breastfeeding, and indicate that women who initiate and continue to breastfeed are older, married, better educated, and have higher family incomes than women who do not breastfeed (Callen and Pinelli, 2004).

For heavy metals, negative associations were also found between SES (educational attainment and income) and internal concentrations of lead (Elreedy et al., 1999) and cadmium (McKelvey et al., 2007) in adult populations in the US, and between SES and lead, but not cadmium, in German children aged 3 to 14 (Becker et al., 2008; Kolossa-Gehring et al., 2007). Studies assessing doseresponse relations of lead stipulate that differences in exposure in childhood are responsible for social inequalities in neurodevelopment and cardiovascular reactivity in adulthood (Bellinger, 2008; Gump et al., 2007). Both lead and cadmium are carcinogenic (Staessen et al., 2001) nefrotoxic (Staessen et al., 1996), neurotoxic (Vermeir et al., 2005) and have been associated with fertility problems.

The strong association between current smoking and blood cadmium in our study sample of Flemish adolescents was also found in an adult population in the US and underlines the importance of preventing smoking initiation and promoting smoking cessation campaigns (McKelvey et al., 2007).

Apart from the studies mentioned earlier, published research on social patterns of internal environmental exposures is rather scarce. Most human biomonitoring studies either do not include social indicators or tend to consider SES data only to control for potential confounding. But rather than claiming to have 'controlled for SES', researchers should acknowledge the potentially relevant aspects of SES when interpreting findings of environment and health studies (Braveman et al., 2005; Hu et al., 2007). Using human biomonitoring, our study attempted to address some of the weaknesses in previous environmental justice studies. First, we used individual-level indicators of exposure to environmental pollution and of socioeconomic status. No aggregated data were used, thereby avoiding results prone to the ecological fallacy. Second, we used biomedical indicators of the actual internal body concentrations of different pollutants, rather than the more generally used geographical measures of external exposure that are considered potential proxies from different points in the source-exposure chain. Third, our study size $(n=1642)$ is considerably higher than most although not all - other research conducted a social stratification of HBM-results, and is representative of the general population rather than for specific medical or social groups such as 
patients with pancreatic cancer (Porta et al., 2008) or pregnant Afro-American woman (Borrell et al., 2004).

Nonetheless, the limitations of the research need to be recognised. In our study, SES was based on a single indicator: the highest education of the adolescents' parents. SES is however a multifaceted concept that cannot be captured with a single indicator (Braveman et al., 2005; Galobardes et al., 2006). Moreover, it has been shown that the type of SES indicator can determine the possible health outcomes and environmental exposures in children and adolescents (Hanson and Chen, 2007; Hoffmann et al., 2009). We selected parental education however not on an 'ad-hoc' basis. Besides more pragmatic reasons (not all SES proxies were adequately measured in the questionnaires), we based SES on parental education because education is termed as a particularly consistent and commonly used indicator in environmental health research and epidemiology (Galobardes et al., 2006; O'Neill et al., 2003). Specifically for children's environmental health, parental education is a recommended individual-level measure of children's SES (Bolte et al., 2005; Carozza et al., 2010). Studies have confirmed that the health and welfare of children are linked to the educational level of their parents, with parental education often a stronger predictor of children's health than family income (Zill, 1996). In line with the theory of Hyman (Hyman et al., 1975) which states that education produces large, pervasive, and enduring effects on knowledge and receptivity to knowledge, we believe that parental education is associated with health-related knowledge and behaviour that affects the exposure to environmental pollution of children and adolescents, for example smoking tobacco products, specific dietary choices and awareness of and access to (preventive) health care. It should however be noted that our study, as well as the greater part of health inequality research, only uses objective measurements of SES (based on parents' material characteristics). Recently however, the development of an adolescent-specific measure of subjective social status, based on adolescents' perceptions of their familial placement in the social hierarchy and their personal placement in the school community, have shown interesting associations with (self-rated) health in the US (Goodman et al., 2001) and Europe (Karvonen and Rahkonen, 2011). This subjective measurement can be a meaningful alternative approach to conceptualise the SES of adolescents, since it embodies an element of social comparison to the association between SES and health. This social comparison has shown to be an important determinant of health inequalities (Wilkinson and Pickett, 2006).

It should also be noted that the parental educational level of our study sample is higher compared to the general Flemish population, and indicates that participating in environmental health research, especially when it is as invasive as human biomonitoring, follows in itself a positive social gradient. 
This has also been observed in other studies (Porta et al., 2009; Tjonneland et al., 2007). Although extensive research on the cultural, social and psychological factors of study participation among socially disadvantaged populations is still missing, it is becoming clear that recruitment of this target group benefits from a more personal approach with a face-to-face relation between researchers and study subjects. This approach can enhance mutual trust and understanding which is essential for involving socially vulnerable populations. When those populations are living in high-risk areas facing multiple environmental stressors, for instance around an industrial hotspot, a continuous human biomonitoring through a community-based participatory approach can be a powerful political tool to address local environmental justice issues, since this may not only provide valuable information for cumulative risk assessments, but may also empower the affected community and engender more effective prevention and intervention actions (NEJAC, 2004; O'Fallon and Dearry, 2002). This touches however also on ethical aspects because a more individual or community-based approach to human biomonitoring may not always match the more traditional medical and clinical ethics which focus on the protection of the autonomy and privacy of research participants (Dumez et al., 2007; Merlo et al., 2008).

Although our results do not favour the traditional environmental justice hypothesis, we believe it remains important to consider the chemical environment in relation to the social environment when monitoring environmental health risks. It seems plausible that issues of environmental justice manifest themselves more in countries with a higher level of social inequality than in Belgium. However, environmental injustice has been shown to be happening in many different ways, and not finding consistent negative social gradients in internal exposure does not mean that inequality in later health effects will not arise from it. Given the same exposure, social disadvantaged people may be more vulnerable to adverse health effects than the general population. Looking at our study sample, the higher prevalence of asthma and the lower self-reported health of adolescents with lower SES can for example indicate a higher susceptibility for the adverse health impact of (even the same concentration) of environmental exposure than adolescents with a higher SES.

\section{Acknowledgements}

The study was commissioned, financed and steered by the Flemish government (Department of Science, Department of Public Health and Environment, Nature and Energy Department). 


\section{References}

Adler, N.E., Newman, K., 2002. Socioeconomic Disparities In Health: Pathways And Policies Health Affairs 21(2), 60-76.

Axelrad, D.A., Goodman, S., Woodruff, T.J., 2009. PCB body burdens in US women of childbearing age 2001-2002: An evaluation of alternate summary metrics of NHANES data. Environmental Research 109(4), 368-378.

Becker, K., Müssig-Zufika, M., Conrad, A., Lüdecke, A., Schulz, C., Seiwert, M., et al. (2008). German Environmental Survey for Children 2003/06 - GerES IV - Human Biomonitoring Levels of selected substances in blood and urine of children in Germany. Berlin: Federal Environment Agency (Umweltbundesamt), Dessau-Roßlau, Robert Koch-Institut.

Bellinger, D.C., 2008. Lead neurotoxicity and socioeconomic status: Conceptual and analytical issues. Neurotoxicology 29(5), 828-832.

Bolte, G., Kohlhuber, M. (2005). PINCHE Project: Final Report WP5 Socioeconomic Factors. Gelderland Midden: Public Health Services.

Bolte, G., Kohlhuber, M., Weiland, S.K., Zuurbier, M., Stansfeld, S., Heinrich, J., 2005. Socioeconomic factors in EU-funded studies of children's environmental health. European Journal of Epidemiology 20(4), 289-291.

Borrell, L.N., Factor-Litvak, P., Wolff, M.S., Susser, E., Matte, T.D., 2004. Effect of socioeconomic status on exposures to polychlorinated biphenyls (PCBs) and dichlorodiphenyldichloroethylene (DDE) among pregnant African-American women. Archives of Environmental Health 59(5), 250-255.

Braveman, P.A., Cubbin, C., Egerter, S., Chideya, S., Marchi, K.S., Metzler, M., et al., 2005. Socioeconomic status in health research - One size does not fit all. Jama-Journal of the American Medical Association 294(22), 2879-2888.

Briggs, D., Abellan, J.J., Fecht, D., 2008. Environmental inequity in England: Small area associations between socio-economic status and environmental pollution. Soc. Sci. Med. 67(10), 16121629.

Brown, P., 1995. Race, class, and environmental health. A review and systematization of the literature. Environmental Research 69(1), 15-30.

Callen, J., Pinelli, J., 2004. Incidence and duration of breastfeeding for term infants in Canada, United States, Europe, and Australia: A literature review. Birth-Issues in Perinatal Care 31(4), 285292.

Carozza, S.E., Puumala, S.E., Chow, E.J., Fox, E.E., Horel, S., Johnson, K.J., et al., 2010. Parental educational attainment as an indicator of socioeconomic status and risk of childhood cancers. British Journal of Cancer 103(1), 136-142.

Cerna, M., Maly, M., Grabic, R., Batariova, A., Smid, J., Benes, B., 2008. Serum concentrations of indicator PCB congeners in the Czech adult population. Chemosphere 72(8), 1124-1131.

Cole, L.W., Foster, S.R., 2001. From the ground up: Environmental racism and the rise of the environmental justice movement. New York: New York University Press.

Croes, K., Baeyens, W., Bruckers, L., Den Hond, E., Koppen, G., Nelen, V., et al., 2009. Hormone levels and sexual development in Flemish adolescents residing in areas differing in pollution pressure. Int. J. Hyg. Environ. Health. 212(6), 612-625.

Davies, J.E., Cassady, J.C., Edmundso, W.f., Morgade, C., Raffonel, A., 1972. Role of social class in human pesticide pollution. American Journal of Epidemiology 96(5), 334-341.

Demers, A., Ayotte, P., Brisson, J., Dodin, S., Robert, J., Dewailly, E., 2002. Plasma concentrations of polychlorinated biphenyls and the risk of breast cancer: A congener-specific analysis. American Journal of Epidemiology 155(7), 629-635.

Den Hond, E., Govarts, E., Bruckers, L., Schoeters, G., 2009. Determinants of polychlorinated aromatic hydrocarbons in serum in three age classes-Methodological implications for human biomonitoring. Environmental Research 109(4), 495-502. 
Den Hond, E., Roels, H.A., Hoppenbrouwers, K., Nawrot, T., Thijs, L., Vandermeulen, C., et al., 2002. Sexual maturation in relation to polychlorinated aromatic hydrocarbons: Sharpe and Skakkebaek's hypothesis revisited. Environ. Health Perspect. 110(8), 771-776.

Dumez, B., Van Damme, K., Casteleyn, L., 2007. Research on the socio-ethical impact of biomarker use and the communication processes in ECNIS NoE and NewGeneris IP. Int. J. Hyg. Environ. Health. 210(3-4), 263-265.

Elreedy, S., Krieger, N., Ryan, P.B., Sparrow, D., Weiss, S.T., Hu, H., 1999. Relations between individual and neighborhood-based measures of socioeconomic position and bone lead concentrations among community-exposed men - The Normative Aging Study. American Journal of Epidemiology 150(2), 129-141.

Evans, G.W., Kantrowitz, E., 2002. Socioeconomic status and health: The potential role of environmental risk exposure. Annual Review of Public Health 23, 303-331.

Galobardes, B., Shaw, M., Lawlor, D.A., Lynch, J.W., Smith, G.D., 2006. Indicators of socioeconomic position (part 1). J. Epidemiol. Community Health 60(1), 7-12.

Goodman, E., Adler, N.E., Kawachi, I., Frazier, A.L., Huang, B., Colditz, G.A., 2001. Adolescents' perceptions of social status: Development and evaluation of a new indicator. Pediatrics 108(2).

Gump, B.B., Reihman, J., Stewart, P., Lonky, E., Darvill, T., Matthews, K.A., 2007. Blood lead (Pb) levels: A potential environmental mechanism explaining the relation between socioeconomic status and cardiovascular reactivity in children. Health Psychology 26(3), 296-304.

Hanson, M.D., Chen, E., 2007. Socioeconomic status and health behaviors in adolescence: A review of the literature. J. Behav. Med. 30(3), 263-285.

Hipp, J.R., Lakon, C.M., 2010. Social disparities in health: Disproportionate toxicity proximity in minority communities over a decade. Health Place 16(4), 674-683.

Hoffmann, B., Kolahgar, B., Rauchfuss, K., Eberwein, G., Franzen-Reuter, I., Kraft, M., et al., 2009. Childhood social position and associations between environmental exposures and health outcomes. Int. J. Hyg. Environ. Health. 212(2), 146-156.

Hornberg, C., Pauli, A., 2007. Child poverty and environmental justice. Int. J. Hyg. Environ. Health. 210(5), 571-580.

Hu, H., Shih, R., Rothenberg, S., Schwartz, B.S., 2007. The epidemiology of lead toxicity in adults: Measuring dose and consideration of other methodologic issues. Environ. Health Perspect. 115(3), 455-462.

Hyman, H.H., Wright, C.R., Reed, J.S., 1975. The enduring effects of education Chicago: University of Chicago Press.

Jacobson, J.L., Humphrey, H.E.B., Jacobson, S.W., Schantz, S.L., Mullin, M.D., Welch, R., 1989. Determinants of polychlorinated-biphenyls (PCBS), poltbrominated biphenyls (PBBS), and dichlorodiphenyl trichloroethane (DDT) levels in the sera of young-children. American Journal of Public Health 79(10), 1401-1404.

Karvonen, S., Rahkonen, O., 2011. Subjective social status and health in young people. Sociol. Health III. 33(3), 372-383.

Kolossa-Gehring, M., Becker, K., Conrad, A., Ludecke, A., Riedel, S., Seiwert, M., et al., 2007. German Environmental Survey for children (GerES IV) - First results. Int. J. Hyg. Environ. Health. 210(5), 535-540.

Krieger, N., 2001. Theories for social epidemiology in the 21st century: an ecosocial perspective. International Journal of Epidemiology 30(4), 668-677.

McKelvey, W., Gwynn, R.C., Jeffery, N., Kass, D., Thorpe, L.E., Garg, R.K., et al., 2007. A biomonitoring study of lead, cadmium, and mercury in the blood of New York city adults. Environ. Health Perspect. 115(10), 1435-1441.

Merlo, D.F., Vahakangas, K., Knudsen, L.E., 2008. Scientific integrity: critical issues in environmental health research. Environ. Health 7, S1-S9.

Mitchell, G., Dorling, D., 2003. An environmental justice analysis of British air quality. Environment and Planning A 35(5), 909-929. 
Morello-Frosch, R., Shenassa, E.D., 2006. The environmental "Riskscape" and social inequality: Implications for explaining maternal and child health disparities. Environ. Health Perspect. 114(8), 1150-1153.

NEJAC. (2004). Ensuring risk reduction in communities with multiple stressors: Environmental justice and cumulative risks/impacts: National Environmmental Justice Advisory Council.

O'Fallon, L.R., Dearry, A., 2002. Community-based participatory research as a tool to advance environmental health sciences. Environ. Health Perspect. 110, 155-159.

O'Neill, M.S., Jerrett, M., Kawachi, L., Levy, J.L., Cohen, A.J., Gouveia, N., et al., 2003. Health, wealth, and air pollution: Advancing theory and methods. Environ. Health Perspect. 111(16), 18611870.

O'Neill, M.S., McMichael, A.J., Schwartz, J., Wartenberg, D., 2007. Poverty, Environment, and Health The Role of Environmental Epidemiology and Environmental Epidemiologists. Epidemiology 18(6), 664-668.

Osius, N., Karmaus, W., Kruse, H., Witten, J., 1999. Exposure to polychlorinated biphenyls and levels of thyroid hormones in children. Environ. Health Perspect. 107(10), 843-849.

Pope, C.A., Burnett, R.T., Thun, M.J., Calle, E.E., Krewski, D., Ito, K., et al., 2002. Lung cancer, cardiopulmonary mortality, and long-term exposure to fine particulate air pollution. JamaJournal of the American Medical Association 287(9), 1132-1141.

Porta, M., de Basea, M.B., Benavides, F.G., Lopez, T., Fernandez, E., Marco, E., et al., 2008. Differences in serum concentrations of organochlorine compounds by occupational social class in pancreatic cancer. Environmental Research 108(3), 370-379.

Porta, M., Gasull, M., Puigdomenech, E., Rodriguez-Sanz, M., Pumarega, J., Rebato, C., et al., 2009. Sociodemographic factors influencing participation in the Barcelona Health Survey study on serum concentrations of persistent organic pollutants. Chemosphere 76(2), 216-225.

Schroijen, C., Baeyens, W., Schoeters, G., Den Hond, E., Koppen, G., Bruckers, L., et al., 2008. Internal exposure to pollutants measured in blood and urine of Flemish adolescents in function of area of residence. Chemosphere 71(7), 1317-1325.

Sexton, K., 1997. Sociodemographic aspects of human susceptibility to toxic chemicals: Do class and race matter for realistic risk assessment? Environmental Toxicology and Pharmacology 4(34), 261-269.

Staessen, J.A., Buchet, J.P., Ginucchio, G., Lauwerys, R.R., Lijnen, P., Roels, H.A., et al., 1996. Public health implications of environmental exposure to cadmium and lead: an overview of epidemiological studies in Belgium. J Cardiovasc Risk 3, 26-41.

Staessen, J.A., Nawrot, T., Den Hond, E., Thijs, L., Fagard, R., Hoppenbrouwers, K., et al., 2001. Renal function, cytogenetic measurements, and sexual development in adolescents in relation to environmental pollutants: a feasibility study of biomarkers. Lancet 357(9269), 1660-1669.

Tjonneland, A., Olsen, A., Boll, K., Stripp, C., Christensen, J., Engholm, G., et al., 2007. Study design, exposure variables, and socioeconomic determinants of participation in diet, cancer and health: A population-based prospective cohort study of 57,053 men and women in Denmark. Scandinavian Journal of Public Health 35(4), 432-441.

Van den Heuvell, R.L., Koppen, G., Staessen, J.A., Den Hond, E., Verheyen, G., Nawrot, T.S., et al., 2002. Immunologic biomarkers in relation to exposure markers of PCBs and dioxins in Flemish adolescents (Belgium). Environ. Health Perspect. 110(6), 595-600.

Vermeir, G., Viaene, M., Staessen, J., Den Hond, E., Roels, H.A., 2005. Neurobehavioural investigations in adolescents exposed to environmental pollutants. Environmental Toxicology and Pharmacology 19(3), 707-713.

Vrijheid, M., Martinez, D., Aguilera, I., Ballester, F., Basterrechea, M., Esplugues, A., et al., 2010. Socioeconomic status and exposure to multiple environmental pollutants during pregnancy: evidence for environmental inequity? J Epidemiol Community Health.

Walker, G., 2009. Beyond Distribution and Proximity: Exploring the Multiple Spatialities of Environmental Justice. Antipode 41(4), 614-636. 
Walker, G., Burningham, K., 2011. Flood risk, vulnerability and environmental justice: Evidence and evaluation of inequality in a UK context. Crit. Soc. Policy 31(2), 216-240.

Wheeler, B.W., Ben-Shlomo, Y., 2005. Environmental equity, air quality, socioeconomic status, and respiratory health: a linkage analysis of routine data from the Health Survey for England. J. Epidemiol. Community Health 59(11), 948-954.

Wilkinson, R.G., Marmot, M., 2003. Social Determinants of Health: The Solid Facts. Copenhagen Denmark: World Health Organization Regional Office for Europe.

Wilkinson, R.G., Pickett, K.E., 2006. Income inequality and population health: A review and explanation of the evidence. Soc. Sci. Med. 62(7), 1768-1784.

Zill, N., 1996. Parental schooling and children's health. Public Health Reports 111(1), 34-43. 